\title{
Combining a Tablet and an Electronic Stethoscope to Create a New Interaction Paradigm for Teaching Cardiac Auscultation
}

\author{
Daniel Pereira ${ }^{1}$, Pedro Gomes ${ }^{1}$, Élodie Mota ${ }^{2}$, Emília Costa $^{2}$, Ricardo Cruz-Correia ${ }^{3,4}$, \\ and Miguel Coimbra ${ }^{1}$ \\ ${ }^{1}$ Instituto de Telecomunicações, Faculdade de Ciências da Universidade do Porto, Portugal \\ ${ }^{2}$ Faculty of Fine Arts of the University of Porto, Portugal \\ ${ }^{3}$ CINTESIS - Center for research in health technologies and information systems \\ ${ }^{4}$ Faculty of Medicine of the University of Porto, Portugal \\ \{danielpereira, rcorreia\} @med.up.pt, \\ \{ptmgomes, mcoimbra\} adcc.fc.up.pt, \\ \{elodie.atom, emilia.d.costa\} egmail.com
}

\begin{abstract}
Learning cardiac auscultation is a fundamental but hard task for a medicine student, involving a combination of gesture mechanics and cardiac sounds interpretation. We aim to create a low cost simulator combining a tablet and an electronic stethoscope, thus defining a new interaction paradigm that al-lows a student to train auscultation when and where they want. In this paper we evaluate the usability of a first approach to this new paradigm using a high-fidelity prototype and its heuristic evaluation.
\end{abstract}

Keywords: Teaching, Heart Auscultation, User interface interaction, Electronic stethoscope.

\section{Motivation}

Cardiac auscultation is the difficult art of listening to heart sounds gathered via a stethoscope, which is routinely used for screening or diagnosis purposes. It requires not only interpretation skills (ex: murmur detection and characterization) but also mechanical ones (ex: where the stethoscope should be placed in the patient's chest). St Clair [1] and Mangione [2] have shown that the ability of medical students to recognize common cardiac murmurs is poor (approximately 20\%) using traditional methods of learning. Barret [3] highlights the importance of repetition for effectively learning auscultation but all simulators that train both interpretation and mechanical skills are too expensive [4].

\section{$2 \quad$ Aim}

We aim to exploit the potential of the touch-based interaction offered by currently popular tablets to develop a cheap cardiac auscultation simulator for medical students, 


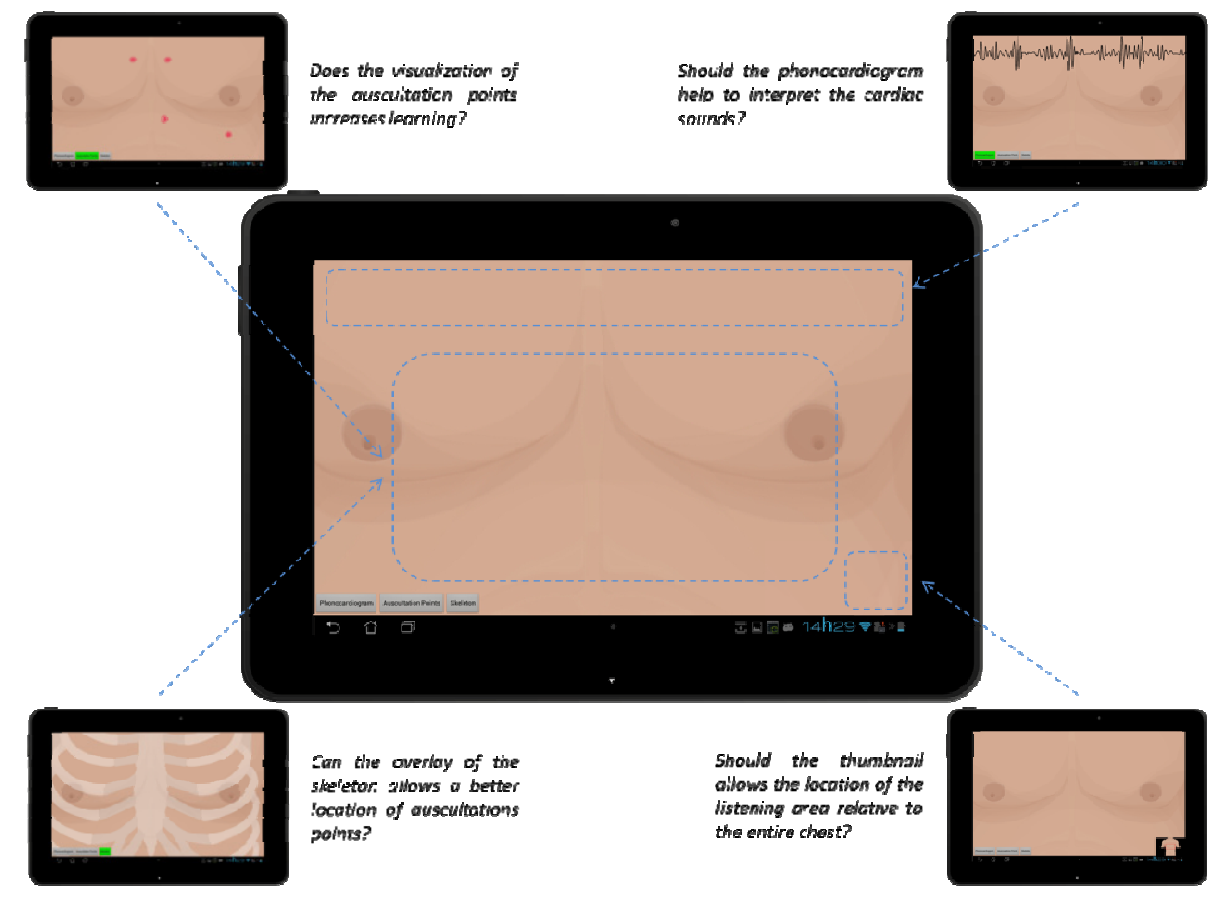

Fig. 1. Preview of the application showing the principal area of auscultation and some functionalities that we will explore

enabling them to train the mechanic and interpretation skills required by auscultation, combining it with a commercial off the shelf electronic stethoscope.

\section{Prototype}

Although developing a full medical student training system requires a multitude of disciplines (hardware, software engineering, pedagogy, etc.), in this paper we will focus on the interactive auscultation functionality. As such, we propose a vertical prototype for a 10 inches tablet that can be used for auscultation of the four principal cardiac focuses (aortic, pulmonic, tricuspid, mitral), combined with a wireless electronic stethoscope as its input/output interaction device.

In Figure 1, we propose a preview of the application and some functionalities that we will explore. The image A in Figure 2 shows the prototype who can allow an interaction using an electronic stethoscope and a high fidelity reproduction of cardiac sounds. The images B and C in Figure 2 show the adjustment made to the stethoscope to allow interaction with the tablet. This adaption was made using aluminum foil and making a slight cut in the plastic diaphragm to allow the passage of a contact point in aluminum in order to conducting electricity through the stethoscope chest piece. 

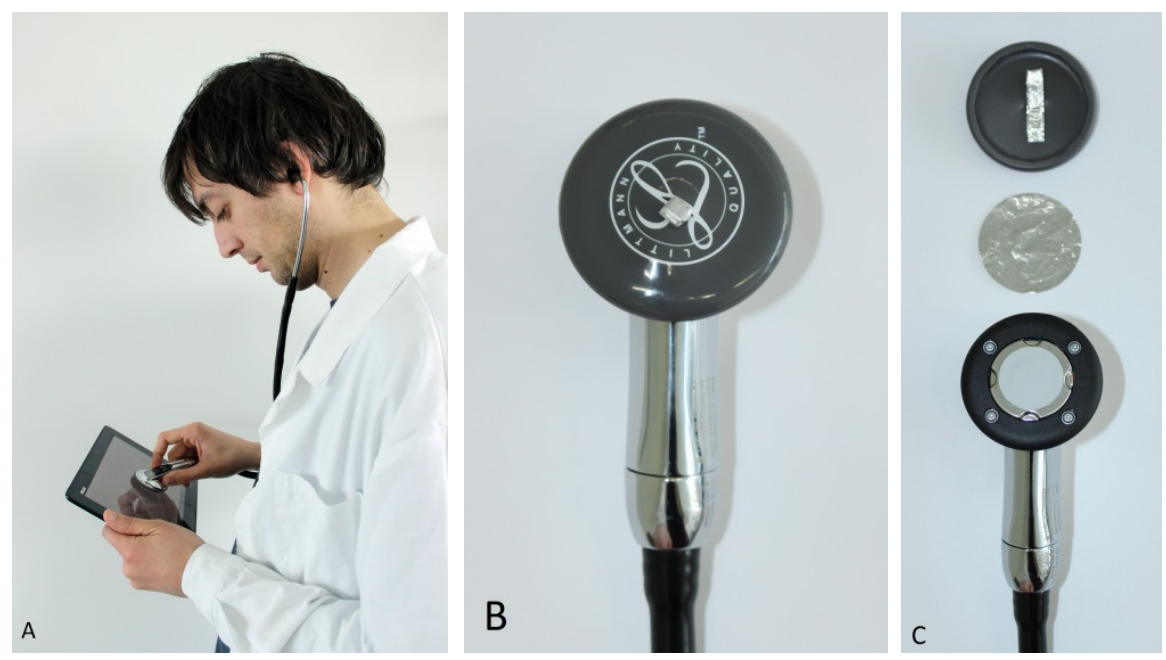

Fig. 2. The first image shows a medical student listening a cardiac sound using an electronic stethoscope to interact with the tablet. The second and third image shows the adjustment made to the stethoscope to allow interaction with the tablet.

\section{Evaluation}

We performed a heuristic evaluation following the principles proposed by Nielsen $[5,6]$. We chose three senior students of the Faculty of Medicine of the University of Porto as evaluators because they represent the target audience of this project. The set of tasks performed by the evaluators is listed in Table 1 . We developed a high-fidelity prototype intended to test the interaction paradigm between these two devices (tablet, stethoscope). The usability and pedagogical impact of a full interactive application intended to teach auscultation is not addressed in this paper.

Results highlighted four errors (Table 2). Three of them are related to the flexibility and efficiency of use and one to the visual clarity of the displayed information. All errors were easy to correct.

Table 1. Taks performed by the evaluators

\begin{tabular}{cl}
\hline $\mathrm{N}^{\mathrm{o}}$ & \multicolumn{1}{c}{ Task } \\
\hline 1 & Enable the display of auscultation points \\
2 & Enable visualization of the skeleton \\
3 & Enable the visualization of phonocardiogram \\
4 & Zooming in on the chest in order to view a large area plan \\
5 & Zooming out of the chest to view the entire chest \\
6 & After zooming in, slide by the image \\
7 & Auscultate the chest with a stethoscope in order to hear a sound \\
\hline
\end{tabular}


Table 2. Detected problems with the heuristic evaluation

\begin{tabular}{|c|c|c|c|}
\hline $\mathrm{N}^{\mathrm{o}}$ & Heuristic & Severity & Description and Possible Solution \\
\hline 1 & $\begin{array}{l}\text { Flexibility and } \\
\text { Efficiency of Use }\end{array}$ & 3 & $\begin{array}{l}\text { The action to zoom in and zoom out is } \\
\text { not obvious. It is suggested that it is possi- } \\
\text { ble to use the open pinch gesture. Another } \\
\text { suggestion is to put a button to the zoom } \\
\text { action. }\end{array}$ \\
\hline 2 & $\begin{array}{l}\text { Match Between } \\
\text { System and the } \\
\text { Real World }\end{array}$ & 2 & $\begin{array}{l}\text { The swipe is too fast. Decrease the } \\
\text { velocity. }\end{array}$ \\
\hline 3 & $\begin{array}{l}\text { Flexibility and } \\
\text { Efficiency of Use }\end{array}$ & 2 & $\begin{array}{l}\text { The timing of the double click is too } \\
\text { long, which causes unexpected zoom out. } \\
\text { Decrease the timing. }\end{array}$ \\
\hline 4 & $\begin{array}{l}\text { Match Between } \\
\text { System and the } \\
\text { Real World }\end{array}$ & 1 & $\begin{array}{l}\text { The ribs of the skeleton are barely } \\
\text { noticeable making it difficult to find the } \\
\text { inter-costal spaces. Increase the skeleton } \\
\text { contrast. }\end{array}$ \\
\hline
\end{tabular}

\section{Discussion}

The data collected with the heuristic evaluation allowed us to improve the highfidelity prototype. However, we will continue working to improve the interaction between the user and the tablet using the stethoscope to provide a simulator that besides training the ear can also train the mechanics of auscultation.

Acknowledgement. This work was supported by FCT project PTDC/EIACCO/100844/2008.

\section{References}

1. St Clair, E.W., et al.: Assessing housestaff diagnostic skills using a cardiology patient simulator. Ann. Intern. Med. 117(9), 751-756 (1992)

2. Mangione, S., et al.: The teaching and practice of cardiac auscultation during internal medicine and cardiology training. A nationwide survey. Ann. Intern. Med. 119(1), 47-54 (1993)

3. Barrett, M.J., et al.: Mastering cardiac murmurs: the power of repetition. Chest 126(2), 470-475 (2004)

4. Karnath, B., Thornton, W., Frye, A.W.: Teaching and testing physical examination skills without the use of patients. Acad. Med. 77(7), 753 (2002)

5. Molich, R., Nielsen, J.: Improving a human-computer dialogue. Commun. ACM 33(3), 338-348 (1990)

6. Nielsen, J.: Heuristic evaluation. In: Nielsen, J., Mack, R.L. (eds.) Usability Inspection Methods, pp. 25-62. John Wiley \& Sons, Inc., New York (1994) 